\title{
Linewidth enhancement factor and modulation bandwidth of lattice matched 1.5 micron InGaAsN/GaAs quantum well lasers
}

\author{
Woon-Ho Seo ${ }^{\mathrm{a}}$, Canice O'Brien ${ }^{\mathrm{b}}$, John F. Donegan*b, Yoonseok Lee ${ }^{\mathrm{a}}$, and Gil-Ho Kim ${ }^{\mathrm{a}}$ \\ ${ }^{a}$ Department of Electronic and Electrical Engineering, Sungkyunkwan University, 440-746, Korea, \\ ${ }^{\mathrm{b}}$ Semiconductor Photonics Group, Department of Physics, Trinity College Dublin, Dublin 2, Ireland.
}

\begin{abstract}
The linewidth enhancement factors of lattice-matched $1.5 \mu \mathrm{m}$ wavelength InGaNAs/GaAs and InGaAs/InP singlequantum-well structures have been calculated using microscopic theory including many-body effects and a 10x10 effective-mass Hamiltonian. For applications which require high gain and carrier densities, InGaNAs/GaAs quantum wells have a much lower linewidth enhancement factor over a temperature range 300-400 K than InGaAs. The linewidth enhancement factor of InGaNAs is almost independent of both carrier density and temperature compared with InGaAs. The small-signal modulation characteristics of these $1.5 \mu \mathrm{m}$ lattice-matched structures and their temperature dependence have also been calculated. It is found that the maximum bandwidth of the InGaNAs/GaAs quantum well lasers is about 2.3 times larger than that of the $\mathrm{InGaAs} / \mathrm{InP}$ quantum well lasers due to the high differential gain. The slope efficiency for the $3 \mathrm{~dB}$ bandwidth as a function of optical density is twice as large for InGaNAs/GaAs as for InGaAs/InP quantum well lasers.
\end{abstract}

Key-words: InGaAsN materials, Semiconductor quantum wells, linewidth enhancement factor, small signal modulation, bandwidth.

\section{INTRODUCTION}

InGaAsN/GaAs quantum wells (QWs) have recently been shown to be very promising materials systems as active media for long wavelength lasers at $1.3 \mu \mathrm{m}$ and possibly $1.5 \mu \mathrm{m}$ in optical communications systems ${ }^{1}$. The large conduction band offset in the InGaAsN system results in a much greater electron confinement compared with the InGaAsP system and should result in much improved high temperature performance in optimized devices ${ }^{2,3}$. In addition, the large refractive index difference in this AlAs-GaAs based system makes it possible to fabricate monolithic Bragg reflectors with a small number of mirror pairs. For use as practical laser systems, a number of key performance parameters need to be investigated for the InGaAsN system and compared with the standard InGaAsP system in order to be able to best choose the system for its particular application. We have carried out a modeling study of the linewidth enhancement factor (LWEF) and the small signal modulation characteristics as a function of carrier density and temperature. The results show that InGaAsN quantum wells have much superior properties over the InGaAs system which is presently used in communications systems lasers. There remains a problem with the growth of InGaAsN materials and as yet, lattice matched materials at 1.5 microns have not been grown. A preliminary report on our modeling studies has been published. ${ }^{4}$

The LWEF is one of the most important parameters of the semiconductor lasers for many practical applications ${ }^{5,6}$. It describes the carrier induced coupling of the gain change to the refractive index change in the active region of the semiconductor laser. The laser linewidth, modulation-induced wavelength chirp, gain guiding, and sensitivity to feedback are dependent on the LWEF. To explore the optimization conditions, it is very important to investigate the dependencies of the LWEF on the gain medium and structural properties of the lasers. Many successful operations of ridge waveguides ${ }^{7}$ distributed-feedback ${ }^{8}$, and vertical-cavity surface-emitting lasers, ${ }^{9}$ with InGaNAs as active layers have been reported. For high-speed optical communication, direct modulation of the laser diodes is essential. Therefore it is important to investigate the dynamic properties of the laser diodes and to understand the modulation characteristics, especially in this new material system. Presently, many experimental results are reported for lasers in $1.3 \mu \mathrm{m}$ wavelength region and now a major challenge is directed towards the achievement of $1.5 \mu \mathrm{m}$ wavelength lasing.

*jdonegan@tcd.ie; phone 353-1-6081987; fax 353-1-6711759 


\section{THEORETICAL MODEL}

The material parameters for the lattice-matched 1.5 micron InGaAsN quantum wells are determined using the band anticrossing model and simple interpolations using known experimental results. Figure 1 shows the bandgap dependence with nitrogen mole fraction for various In alloy concentrations. The bandgap energy in the quaternary system is given by:

$$
\begin{aligned}
E_{g}(x, y) & =x y E_{g}(\operatorname{InN})+x(1-y) E_{g}(\operatorname{InAs}) \\
& +(1-x) y E_{g}(G a N)+(1-x)(1-y) E_{g}(G a A s) \\
& -x(1-x)\left[y C_{\text {InGaN }}+(1-y) C_{\text {InGaAs }}\right] \\
& -y(1-y)\left[x C_{\text {InNAs }}+(1-x) C_{\text {GaNAS }}\right]
\end{aligned}
$$

Within the band anti-crossing model, although there is no direct coupling between the nitrogen band and the valence bands, there is an indirect interaction through the coupling with the conduction band ${ }^{10-12}$. A $10 \times 10$ effective mass Hamiltonian is used for energy band structure calculations of an InGaNAs/GaAs QW including the conduction-valence interaction with the spin degenerate nitrogen band. In the free carrier model with simple phenomenological relaxation time approximation, the LWEF is overestimated and it is underestimated in the model which includes band gap renormalization but ignores the Coulomb enhancement effect. ${ }^{13}$

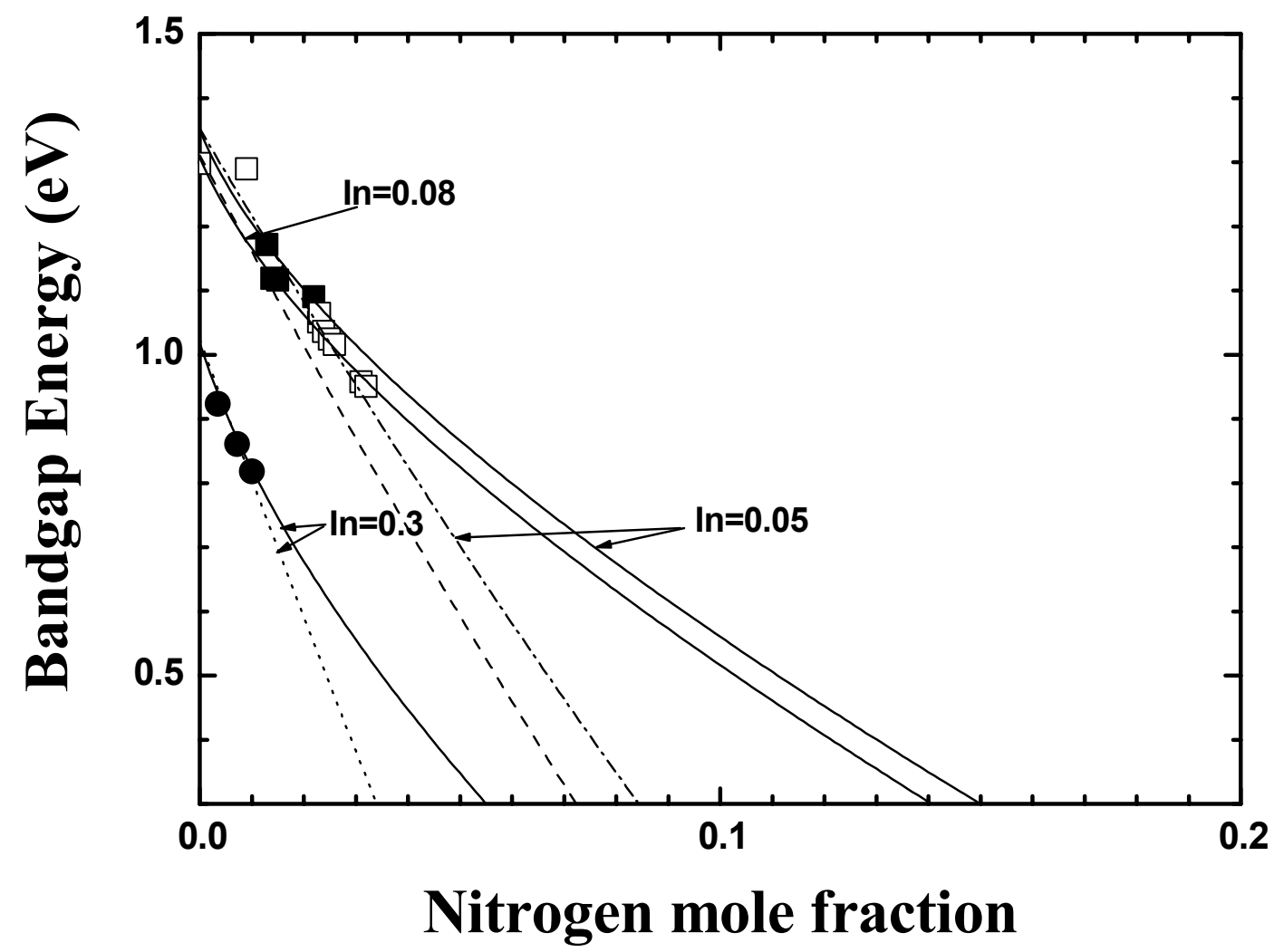

Figure 1. The bandgap energy dependence of the quantum well material with nitrogen concentration at various In concentrations. The solid lines are the band anti-crossing model and the dashed lines, the interpolation from the experimental data. 
In this work, we employ a microscopic theory based on the semiconductor Bloch equations with Coulomb interaction and band gap renormalization. This model is used for the calculation of optical gain and refractive index changes. ${ }^{14}$ Carrier-carrier scattering is treated at the level of the quantum kinetic regime instead of using simple phenomenological parameters for the line broadening, ${ }^{15}$ According to the band anti-crossing model, the electron-nitrogen coupling of $\mathrm{In}_{\mathrm{x}} \mathrm{Ga}_{1-\mathrm{x}} \mathrm{N}_{\mathrm{y}} \mathrm{As}_{1-\mathrm{y}}$ is described by the matrix:

$$
\left(\begin{array}{cc}
E_{0}(x, y, T)+\frac{\hbar^{2} k^{2}}{2 m^{*}(x)} & V_{N M}(y) \\
V_{N M}(y) & E_{N}(x, y)
\end{array}\right)
$$

where $m^{*}(x)$ is the bulk electron effective mass of $\operatorname{In}_{x} \mathrm{Ga}_{1-x} \mathrm{As}$. The conduction band energy $E_{0}(x, y, T)$ is given by $E_{0}(x, y, T)=E^{0}(x, T=0)-1.55 y-\frac{\alpha T^{2}}{\beta+T}(\mathrm{eV})$ Considering the indium concentration effect, the nitrogen energy level is taken as ${ }^{12} E_{N}(x, y)=1.65-2.52 y-0.4 x(1-x)(e V)$ and the coupling term is $V_{N M}(y)=-3.0 \sqrt{y} \quad(\mathrm{eV})$. Following the fitting of the experimental results with various indium and nitrogen mole fractions, the Varshni coefficients $\alpha$ and $\beta$ are determined which can be used for the temperature dependent bandgap determination. For our modeling study, we choose a $7 \mathrm{~nm} \mathrm{In}_{0.53} \mathrm{Ga}_{0.47} \mathrm{As} / \mathrm{InP}$ and $\mathrm{In}_{0.1} \mathrm{Ga}_{0.9} \mathrm{~N}_{0.036} \mathrm{As}_{0.964} / \mathrm{GaAs} \mathrm{QWs}$ with $1.5 \mu \mathrm{m}$ emission. The lattice matched condition is obtained by the application of the band anti-crossing model and Vergard's law for the known experimental results. ${ }^{12,16,17}$ The calculated gain spectra in the two quantum well systems are shown in fig. 2.



(a)

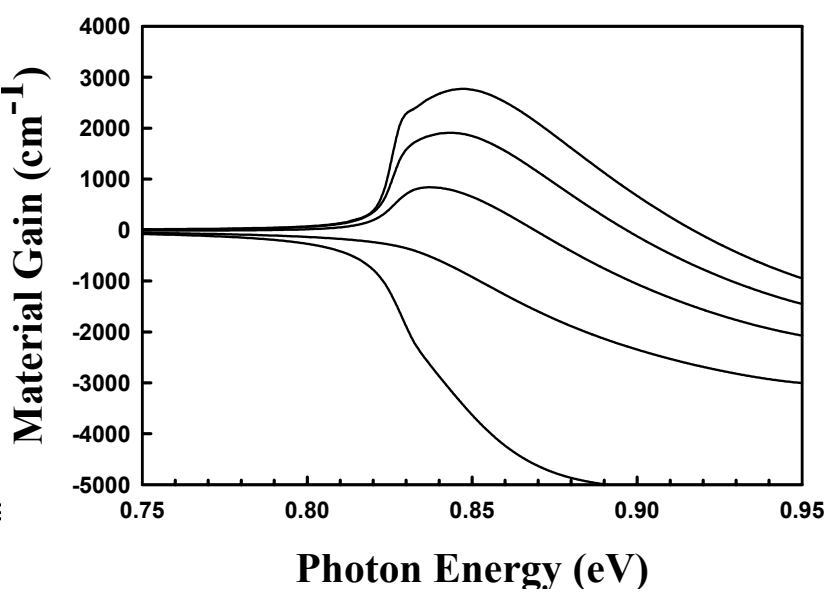

(b)

Figure 2. A comparison of gain in the InGaAs (a) and InGaAsN (b) systems. The gain spectra are calculated for carrier densities of $0.1,1,2,3,4 \times 10^{18} \mathrm{~cm}^{-3}$.

It is clear that there is much higher gain in the InGaAsN system for a particular carrier density. The better match of the carrier effectives masses and therefore the better match of the densities of states in the two bands is responsible for the higher gain in the nitride system. The peak gain of the two systems are presented in Fig. 3 as a function of carrier density. 


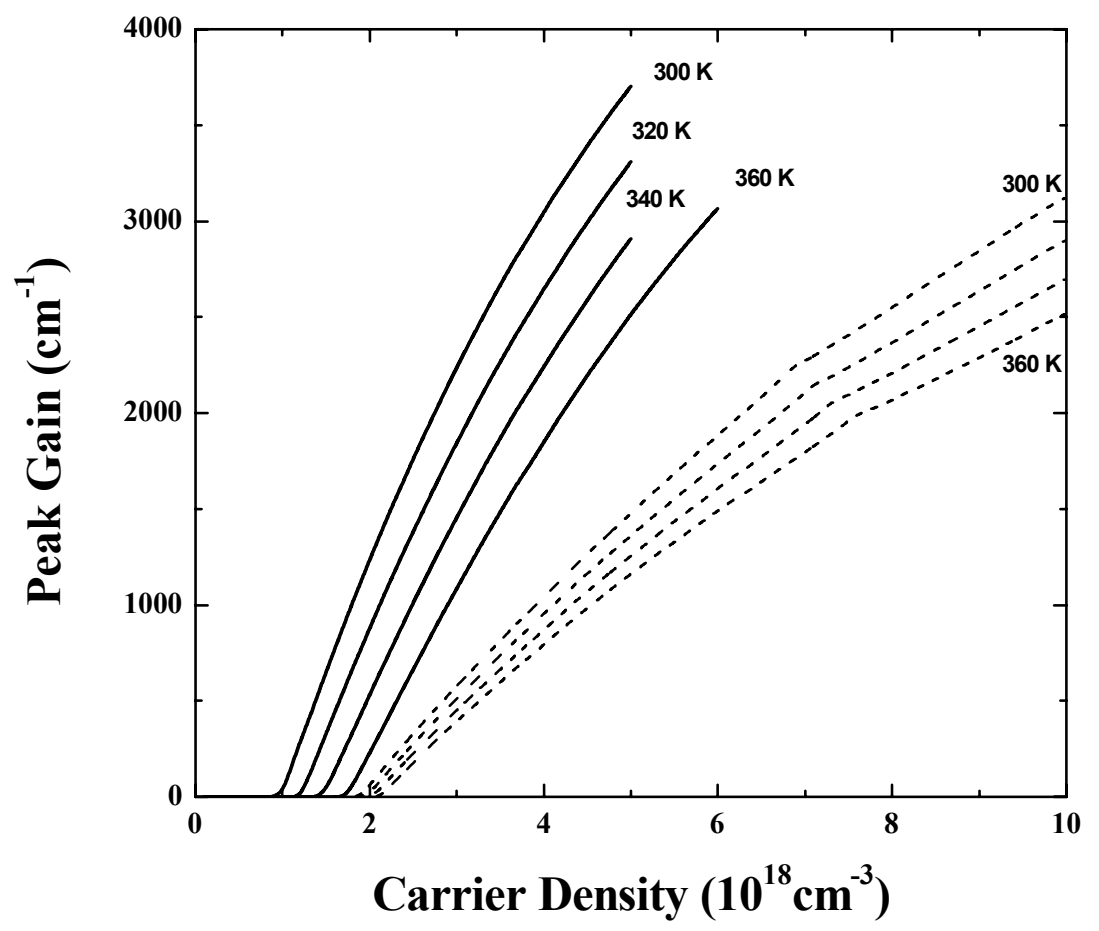

Figure 3. Peak gains as a function of carrier density for the two quantum well systems, solid line InGaAsN, dashed line InGaAs. Data are presented over the temperature range of 300 to $360 \mathrm{~K}$.

For temperatures below $400 \mathrm{~K}$, the InGaNAs system has the lower transparency carrier density. The differential gain of InGaNAs is higher than that of InGaAs and almost insensitive to varying temperatures (see Fig. 7 later). As for the gain, the lower transparency and larger differential gain of InGaNAs are due to the large electron effective mass which is $0.104 m_{0}$ and about 2.4 times larger than that of InGaAs. The better density of states match between conduction band and valence band leads to low values of transparency and high differential gain. The large electron effective mass compared with InGaAs materials comes from the strong interaction between the conduction band and a narrow resonant band formed by the nitrogen states. ${ }^{10}$ Using lattice mismatching in the system, the electron and hole effective mass can be changed which leads to changes in threshold conditions and differential gain. Also the increase of nitrogen mole fraction enhances the electron effective mass. Therefore, by adjusting the indium and nitrogen mole fractions in the quaternary material, the matching between the two density of states can be improved resulting in better optical properties.

\section{LINEWIDTH ENHANCEMENT FACTOR}

For high-speed modulation of lasers in fiber-optic communication system, a low LWEF with low temperature and carrier density dependencies are required. A low LWEF reduces pulse chirp when compared with the InGaAs system allowing higher speed modulation and therefore higher speed communications. The carrier density dependence of the LWEF and the temperature dependence of LWEF at certain carrier densities are shown in Fig. 4. All LWEFs are obtained at the peak gain positions. 


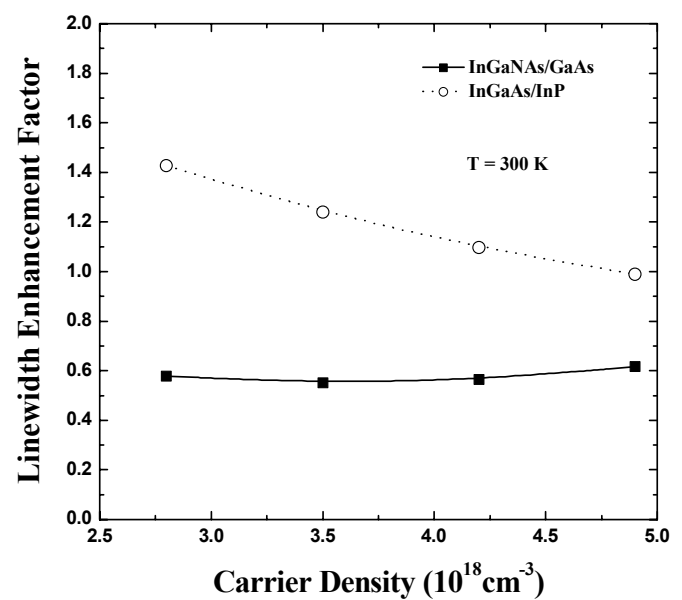

(a)



(b)

Figure 4. (a) The linewidth enhancement factor versus carrier density (b) The linewidth enhancement factor versus temperature at a fixed carrier density of $3.5 \times 10^{18} \mathrm{~cm}^{-3}$.

For the whole carrier density range of the figure in Fig. 4(a), the LWEF of InGaNAs is lower than that of InGaAs and is almost constant with carrier density. This independence of the LWEF on carrier density is particularly valuable as it results in low pulse chirp at high modulation frequencies. This is a direct result of high differential gain as shown in Fig. 3. Figure 4(b) shows the temperature dependence of LWEF at a carrier density of $3.5 \times 10^{18} \mathrm{~cm}^{-3}$. At a fixed carrier density, the temperature dependence of InGaAs is larger than that of InGaNAs due to the larger differential-gain decrease with increasing temperature. The temperature dependence of the LWEF for InGaAs increases at the elevated carrier densities compared with that for InGaNAs which is almost temperature insensitive.

To compare the thermal characteristics of the LWEF for InGaNAs and InGaAs in real operating environments, the temperature dependence of LWEF at a gain of $2500 \mathrm{~cm}^{-1}$ is shown in Fig. 5. The LWEF for InGaNAs is much lower

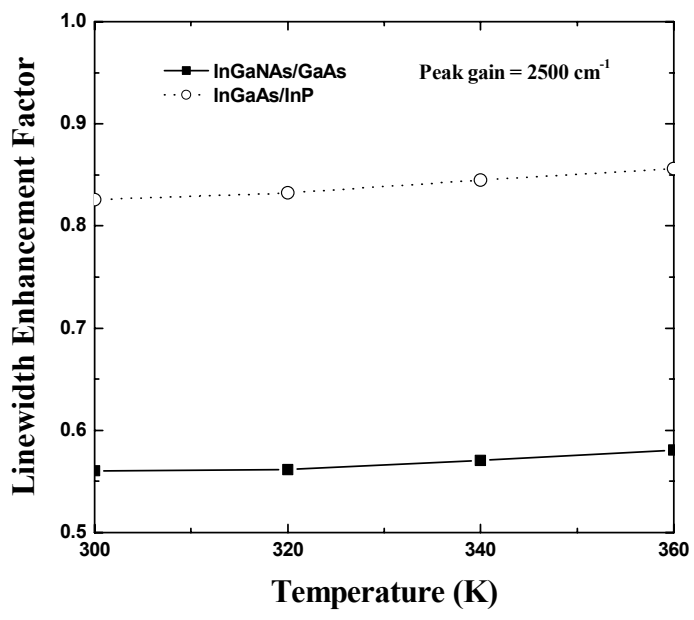

Figure 5. The LWEF as a function of temperature at a peak gain of $2500 \mathrm{~cm}^{-1}$. 
than that for InGaAs and for the temperatures between 300 and $360 \mathrm{~K}$, the relative change of LWEF for InGaNAs is smaller than that for InGaAs by a factor or 2 . To obtain a gain of $2500 \mathrm{~cm}^{-1}$, a higher carrier density is needed for the InGaAs system which results in a reduction of the differential gain as shown in Fig. 3. Due to the larger difference of the conduction band and valence band effective masses for InGaAs, it requires more carriers to achieve the same gain compared with InGaNAs. In high temperature and high electric field environments for device applications, the large conduction band offset and electron effective mass of InGaNAs make this material a strong candidate for high-speed devices.

\section{MODULATION CHARACTERISTICS}

Using rate equations and nonlinear gain saturation, the small signal frequency response function is given by:

$$
R_{\text {mod }}(\omega)=\left(\frac{\Gamma \tau_{p}}{q d}\right) \frac{\omega_{r}^{2}}{\sqrt{\left(\omega^{2}-\omega_{r}^{2}\right)^{2}+\omega^{2} \gamma^{2}}}
$$

where the damping factor is defined as

$$
\gamma=K f_{r}^{2}+\frac{1}{\tau} \text { and the } K \text { factor is given by: } K=4 \pi^{2}\left(\tau_{p}+\frac{\varepsilon}{v g^{\prime}}\right)
$$

where $\tau_{\mathrm{p},}, \tau, f_{r}, g^{\prime}, \Gamma$, and $\varepsilon$ are the photon lifetime, carrier lifetime, resonant frequency, differential gain, optical confinement factor, gain suppression coefficient, and the frequency $\omega_{r}=2 \pi f_{r}$, respectively ${ }^{18}$.

In the numerical calculations, $\tau_{\mathrm{p},}, \tau, \Gamma$, and $\varepsilon$ are set equal to $1.5 \mathrm{ps}, 1.0 \mathrm{~ns}, 0.1$, and $3.0 \times 10^{-23} \mathrm{~m}^{3}$, respectively for both material systems. For the first three parameters, this is a very good approximation and we will discuss the $\varepsilon$ parameter further below. Fig.6 shows the small-signal frequency response of InGaNAs/GaAs and InGaAs/InP QW lasers with different optical densities at a temperature of $300 \mathrm{~K}$. At the same optical density, the bandwidth of InGaNAs/GaAs QW

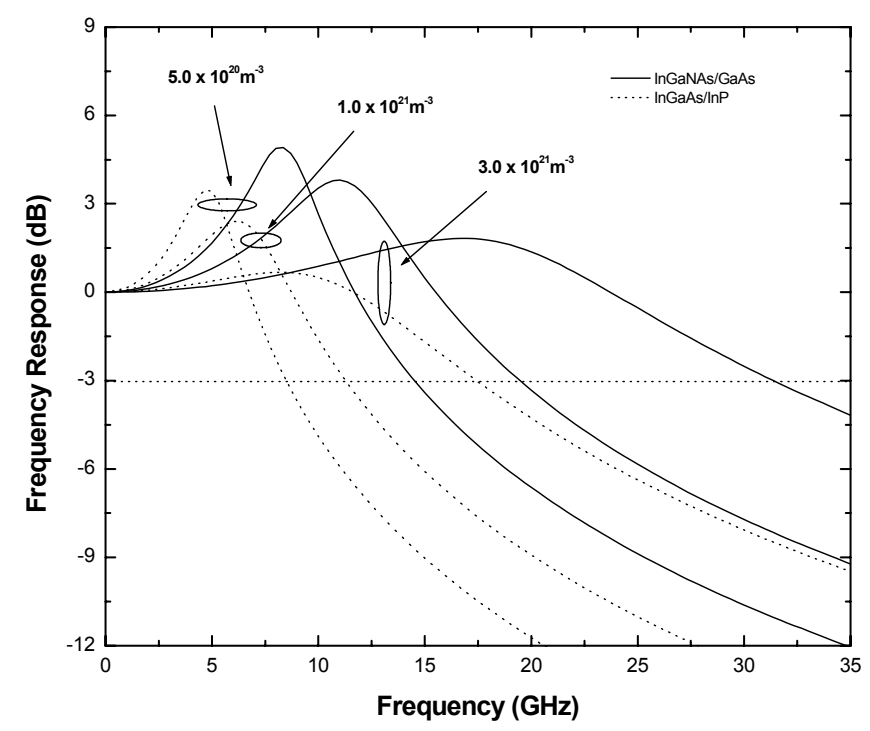

Figure 6. Frequency responses of InGaNAs/GaAs QW (solid lines) and InGaAs/InP QW (dotted lines) at a temperature of $300 \mathrm{~K}$ for various optical densities. 
lasers is larger than that of InGaAs/InP QW lasers and also the amount of increase with the optical density increase is enhanced in the former system. The differential gains of the two systems are presented in fig.7 as a function of temperature at a peak gain of $2500 \mathrm{~cm}^{-1}$. Once again, it is clear that the InGaAsN system has a much greater differential gain, although it decreases more with temperature than InGaAs. In fig. 8(a) the temperature dependence of K-factors for

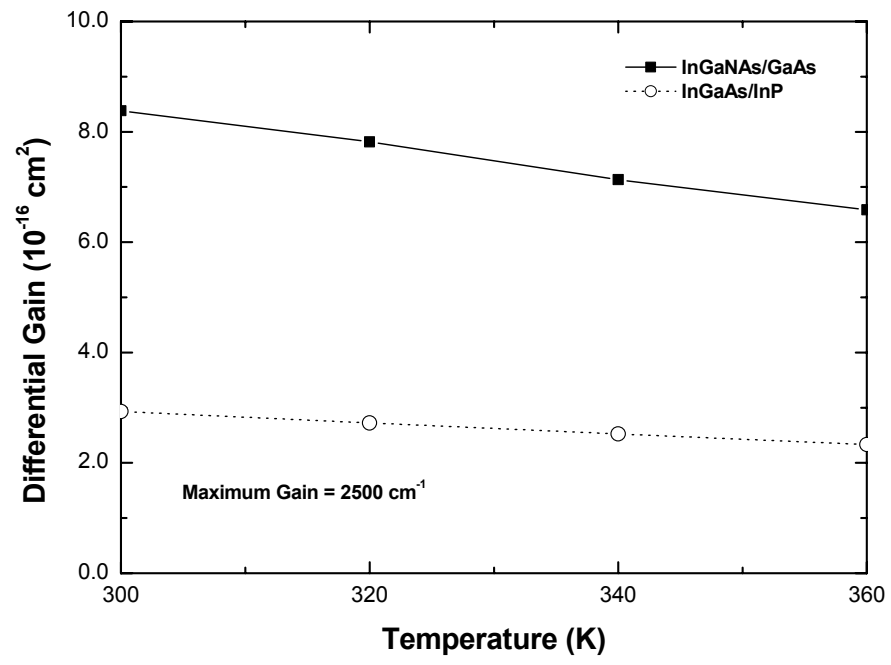

Figure 7. Temperature dependence of differential gains for InGaNAs/GaAs QW (solid line with solid squares) and InGaAs/InP QW (dotted line with open circles) at a peak gain of $2500 \mathrm{~cm}^{-1}$

both InGaNAs/GaAs and InGaAs/InP QW lasers are presented. The K-factor of InGaNAs lasers is $0.225 \mathrm{~ns}$ and about 2.5 times smaller than that of InGaAs lasers at a temperature of $300 \mathrm{~K}$ which gives a maximum resonance frequency of 39.4 GHz (16.7 GHz for InGaAs/InP QW lasers.) using the $3 \mathrm{~dB}$ cutoff condition, $f_{r, \max }=\frac{2 \pi \sqrt{2}}{K}$. This is also due to the enhanced differential gain in the InGaNAs system. For the temperature range of $300-360 \mathrm{~K}$, the temperature dependence of the $3 \mathrm{~dB}$ modulation bandwidth of InGaAs/InP QW lasers is smaller than that of InGaNAs/GaAs QW lasers, but the values are much larger for the InGaNAs system as shown in fig. 8(b). Also, the increasing rate of the $3 \mathrm{~dB}$ modulation bandwidth for InGaNAs with the injection current (that is, optical density) is twice as large as that for InGaAs resulting in a higher modulation current efficiency factor (the slope of modulation bandwidth as a function of injection current). The large conduction band offset (deep quantum well) in the InGaNAs/GaAs QW increases the electron escape time which leads to the decrease in damping rate ${ }^{19}$ and gives better high-temperature performance in high-speed modulation. In this paper, the same gain suppression coefficient $\varepsilon$ is used for both systems and it's temperature-independence is assumed as it was shown by the experiments for $1.3 \mu \mathrm{m} \mathrm{InGaAsP/InP} \mathrm{lasers}{ }^{20}$. There are no available experimental results to determine the gain suppression coefficient of InGaNAs material, but the stronger confinement of electrons due to the enhanced conduction band offset should result in a lower gain suppression coefficient as shown by the experiments for InGaAs/InAlGaAs QW lasers in comparison with InGaAs/InGaAsP QW lasers ${ }^{21}$. This would further increase the difference of the modulation bandwidth between the InGaNAs/GaAs and InGaAs/InP QW lasers. It is therefore a reasonable assumption that the $\varepsilon$ coefficient of InGaAsN lasers will be much less than for InGaAsP lasers. Our use of the same value is therefore a cautious approach and the modulation characteristics that will be observed in future devices will likely be even better than those given in this paper. 


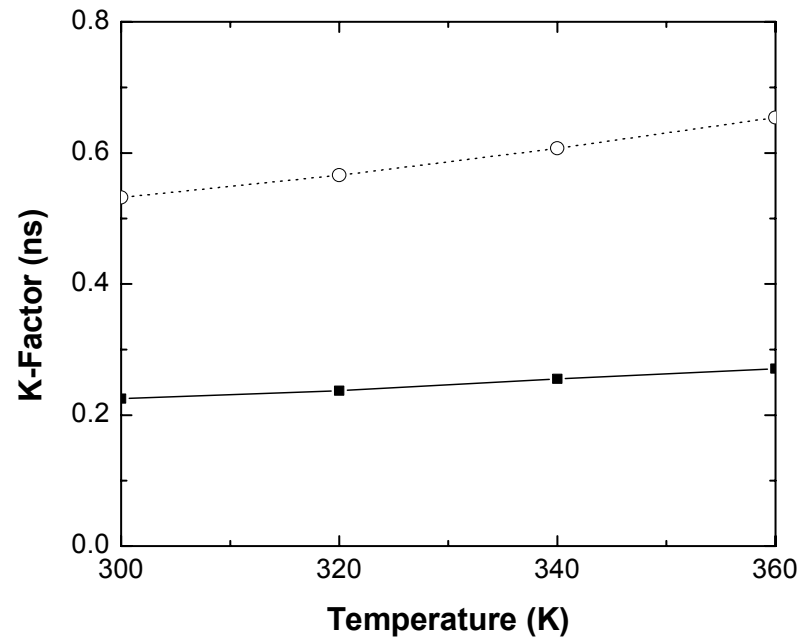

(a)

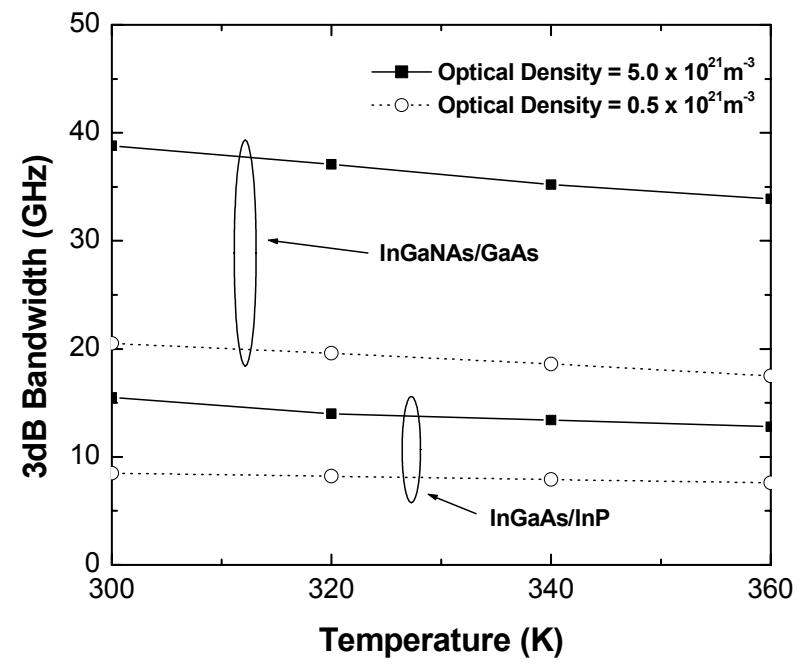

(b)

Figure 8. (a) K-factors as a function of temperature for InGaNAs/GaAs QW (solid line with solid squares) and InGaAs/InP QW (dotted line with open circles.) $\quad$ (b) Temperature dependence of $3 \mathrm{~dB}$ bandwidth for InGaNAs/GaAs and InGaAs/InP QWs at the optical density of $0.5 \times 10^{21} \mathrm{~m}^{-3}$ (solid line with solid squares) and of $5.0 \times 10^{21} \mathrm{~m}^{-3}$ (dotted line with open circles).

\section{CONCLUSIONS}

In conclusion, to allow for a comparison with conventional InGaAs material, the lattice-matched conditions for InGaNAs quantum well materials are determined from experimental results published in the literature. The optical gain, LWEF and their temperature dependence for InGaNAs QWs are calculated with microscopic theory and a 10x10 effective-mass Hamiltonian and a comparison is made with those of InGaAs QWs. It is shown that an InGaNAs/GaAs QW has better characteristics in optical gain and LWEF than InGaAs QWs. The enhanced differential gain and the smaller LWEF of InGaNAs show its potential for application in optical communications. The temperature insensitivity of the LWEF and its clamping with increasing carrier injection in InGaNAs/GaAs QW promise more stable semiconductor lasers which have spectrally and spatially more stable modal properties under high-speed modulation conditions with narrow laser linewidth, reduced filamentation, and low frequency chirp. Also, the larger electron effective mass and conduction band offset of InGaNAs/GaAs QWs, the greater refractive index contrast achievable in GaAs-based systems than conventional InP-based materials make the application of the material for long-wavelength VCSELs possible.

Lattice-matched InGaNAs/GaAs QW lasers have enhanced high-speed modulation characteristics when compared with conventional InGaAs/InP QW lasers for a wavelength of $1.5 \mu \mathrm{m}$. This is mainly due to the larger differential gain in the InGaNAs system resulting again from the larger conduction band effective mass. The maximum $3 \mathrm{~dB}$ modulation bandwidth of $39.4 \mathrm{GHz}$ (2.3 times larger than that of the InGaAs/InP system) is achieved by assuming that a gain suppression coefficient of $3.0 \times 10^{-23} \mathrm{~m}^{3}$ at a temperature of $300 \mathrm{~K}$ for both systems. Using multiple quantum wells and the introduction of the lattice-mismatch in the InGaNAs material system can further improve the modulation characteristics. 


\section{ACKNOWLEDGMENTS}

The authors are grateful to James O'Gorman for valuable discussions and suggestions. This work was supported in Korea by the National Research and Development Project for Nano Science and Technology (contract No. N11-021204-0003) of NOST. The work was supported by the Phase II projects of Enterprise Ireland under the Programmes in Advanced Technologies in Ireland.

\section{REFERENCES}

1. M. Kondow, T. Kitani, S. Nakatsuka, M. C. Larson, K. Nakahara, Y. Yazawa, M. Okai, and K. Uomi, "GaInNAs: a novel material for long-wavelength semiconductor lasers" IEEE J. Sel. Top. Quantum Electron. 3, 719-730, 1997.

2. Z. Pan, L. H. Li, Y. W. Lin, B. Q. Sun, D. S. Jiang, and W. K. Ge, "Conduction band offset and electron effective mass in GaInNAs/GaAs quantum-well structures with low nitrogen concentration” Appl. Phys. Lett. 78, 2217-2219, 2001.

3. C. K. Kim and Y. H. Lee, "Thermal characteristics of optical gain for GaInNAs quantum wells at $1.3 \mu$ m" Appl. Phys. Lett. 79, 3038-3040, 2001.

4. W. H. Seo, J. F. Donegan, "Linewidth enhancement factor of lattice-matched InGaNAs/GaAs quantum wells" Appl. Phys. Lett., 82, 505-507, 2003.

5. L. Olofsson and T. G. Brown, "On the linewidth enhancement factor in semiconductor lasers" Appl. Phys. Lett. 57, 2773-2775, 1990.

6. J. Hader, D. Bossert, J. Stohs, W. W. Chow, S. W. Koch, and J. V. Moloney, "Clamping of the linewidth enhancement factor in narrow quantum-well semiconductor lasers" Appl. Phys. Lett. 74, 2277-2279, 1999.

7. W. Ha, V. Gambin, M. Wistey, S. Bank, S. Kim, and J. S. Harris, Jr., "Multiple-quantum-well GaInNAs-GaNAs ridgewaveguide laser diodes operating out to 1.4 \&mu;m" IEEE Photonics Technol. Lett., 14, 591-593, 2002.

8. M. Reinhardt, M. Fischer, M. Kamp, J. Hofmann, and A. Forchel, "1.3-micron GaInNAs-AlGaAs distributed feedback lasers" IEEE Photonics Technol. Lett., 12, 239-241, 2000.

9. C. W. Coldren, M. C. Larson, S. G. Spruytte, and J. S. Harris, "1200 nm GaAs-based vertical cavity lasers employing GaInNAs multiquantum well active regions" Electron. Lett., 36, 951-952, 2000.

10. W. Shan, W. Walukiewicz, J. W. Ager III, E. E. Haller, J. F. Geisz, D. J. Friedman, J. M. Olson, and S. R. Kurz, "Band Anticrossing in GaInNAs Alloys" Phys. Rev. Lett. 82, 1221-1224, 1999.

11. J. Hader, S. W. Koch, J. V. Moloney, and E. P. O'Reilly, "Gain in $1.3 \mu \mathrm{m}$ materials: InGaNAs and InGaPAs semiconductor quantum-well lasers" Appl. Phys. Lett. 77, 630-632, 2000.

12. W. Shan, W. Walukiewicz, J. W. Ager III, E. E. Haller, J. F. Geisz, D. J. Friedman, J. M. Olson, S. R. Kurz, H. P. Xin, and C. W. Tu, "Band anticrossing in III-N-V alloys" Phys. Status Solidi B 223, 75-85, 2001.

13. W. W. Chow, S. W. Koch, and M. Sargent III, Semiconductor Laser Physics, Springer-Verlag, Berlin, 1994.

14. M. Lindberg and S. W. Koch, "Effective Bloch equations for semiconductors" Phys. Rev. B 38, 3342-3350, 1988.

15. R. Binder, D. Scott, A. E. Paul, M. Lindberg, K. Henneberger, and S. W. Koch, "Carrier-carrier scattering and optical dephasing in highly excited semiconductors" Phys. Rev. B 45, 1107-1115, 1992.

16. R. J. Potter, N. Balkan, X. Marie, H. Carre`re, E. Bedel, and G. Lacoste, "Interaction strength between the highly localised nitrogen states and the extended semiconductor matrix states in GaInNAs"Phys. Status Solidi A 187, 623-632, 2001.

17. T. Hakkarainen, J. Toivonen, M. Sopanen, and H. Lipsanen, "GaInNAs quantum well structures for $1.55 \mu \mathrm{m}$ emission on GaAs by atmospheric pressure metalorganic vapor phase epitaxy” J. Cryst. Growth 234, 631-636, 2002.

18. S. L. Chuang: 'Physics of Optoelectronic Devices' Wiley Interscience, New York, 1995.

19. C. Y. Tsai, F. P. Shih, T. L. Sung, T. Y. Wu, and C. H. Chen, "A small-signal analysis of the modulation response of high-speed quantum-well lasers: effects of spectral hole burning, carrier heating, and carrier diffusion-capture-escape" IEEE J. Quantum Electron., 33, 2084-2096, 1997.

20. M. Ishikawa, T. Fukushima, R. Nagarajan, and J. E. Bowers, "Temperature dependence of damping in high-speed quantum-well lasers" Appl. Phys. Lett., 61, 396-398, 1992.

21. A. Grabmaier, A. Hangleiter, G. Fuchs, J. E. A. Whiteway, and R. W. Glew, "Low nonlinear gain in InGaAs/InGaAlAs separate confinement multiquantum well lasers" Appl. Phys. Lett., 59, 3024-3026, 1991. 